\title{
Lateral Abdominal Wall Reconstruction
}

\author{
Sahil K. Kapur, MD ${ }^{1}$ Charles E. Butler, MD, FACS ${ }^{1}$ \\ ${ }^{1}$ Department of Plastic Surgery, University of Texas MD Anderson \\ Cancer Center, Houston, Texas \\ Semin Plast Surg 2018;32:141-146.
}

\begin{abstract}
Address for correspondence Sahil K. Kapur, MD, Assistant Professor, Department of Plastic Surgery, University of Texas MD Anderson Cancer Center, 1400 Pressler St Unit 1488, Houston, TX 77030-4009 (e-mail: skkapur@mdanderson.org).
\end{abstract}

\begin{abstract}
Keywords

- flank hernia

- pillar-anchored repair

- bioprosthetic mesh

- lateral abdominal wall reconstruction

Lateral abdominal wall defects, while rare, present a more challenging problem than commonly encountered ventral defects due to the complexity of the anatomy, physiologic forces, and impact of muscle denervation. The lateral abdominal wall encompasses a large surface area ranging from the costal margin superiorly to the iliac crest inferiorly and from the linea semilunaris anteriorly to the paraspinous musculature posteriorly. The ratio of muscle to fascia/aponeurosis is much higher, which makes repair through muscle tissue versus fascia less secure. Furthermore, these defects are subject to asymmetric forces caused by the independent contraction of anterior and posterior muscle units, which lead to unbalanced strain and hernia progression. These features necessitate the use of wide underlay mesh load bearing repairs supported by the static pillars of the abdominal wall. Management can be further complicated when defects extend beyond the defined boundaries, requiring surgical repair to be adapted based on the border structures involved. Primary fascial coaptation may not be as easily accomplished, and therefore careful planning is important to ensure stable coverage of exposed mesh.
\end{abstract}

Lateral abdominal wall defects are encountered much less frequently than ventral abdominal wall defects. They vary significantly in their anatomy and behavior and therefore need to be managed differently. While these defects are rare, they can lead to significant comorbidity for the patient. The main etiology is surgical or iatrogenic trauma involving resection of tissue or denervation of the local musculature. Congenital defects are very rare and occur less frequently than congenital defects of the ventral abdominal wall (gastroschisis, omphalocele). ${ }^{1}$ Iatrogenic causes result from incisions that lead to disinsertion of part of the oblique muscle complex or denervation of these muscles by disruption of the T7-T12 nerve supply. This overlapping injury pattern is seen with certain vascular and renal access incisions as well as with Kocher and Chevron incisions in hepatobiliary surgery and oncologic resections. ${ }^{2-4}$ These defects can present as hernias caused by disruption of all overlying muscle layers or bulges caused by disruption or denervation of a subset of the muscle layers comprising the lateral abdominal wall. The incidence after retroperitoneal access has been reported to vary from 8 to $57 \%{ }^{5,6}$

Issue Theme Abdominal Wall Reconstruction; Guest Editor: Jesse $C$. Selber, MD, MPH, FACS

\section{Anatomy}

\section{General}

Many of the techniques used in ventral hernia repair have been extrapolated to address lateral defects. However, the anatomy and pathophysiology of these defects are significantly different from ventral defects, and these differences must be taken into account prior to planning repair., ${ }^{7,8}$ The boundaries of the lateral abdominal wall include the costal margin superiorly and the iliac crest inferiorly. It stretches from the paraspinous muscle complex (paraspinous muscles, quadratus lumborum, iliopsoas) posteriorly to the linea semilunaris anteriorly. The components of the lateral abdominal wall include the external oblique muscles, which originate from the lower eight ribs and insert into the linea alba; the internal oblique muscles, which originate from the iliac crest, thoracolumbar fascia, and inguinal ligament and run superomedially to insert into the costal margin and linea abla; and the transversus abdominis muscles, which originate from the costal cartilages of ribs 6 through 12 , thoracolumbar fascia, anterior iliac crest, and inguinal ligament and insert through their aponeurosis into the linea alba.

Copyright (c) 2018 by Thieme Medical Publishers, Inc., 333 Seventh Avenue, New York, NY 10001, USA. Tel: +1(212) 584-4662.
DOI https://doi.org/ 10.1055/s-0038-1666801. ISSN $1535-2188$. 
The ventral abdominal wall, on the other hand, is bounded by the linear semilunaris laterally, the costal margins superiorly, and the pubic bone and medial inguinal ligament inferiorly. It comprises of the rectus muscle complexes that are contained within the anterior and posterior rectus sheaths, which are made up of the aponeuroses of the external oblique, internal oblique, and transversus abdominis muscles. ${ }^{7}$

\section{Boundaries}

Defects involving the lateral abdominal wall commonly extend beyond its established boundaries. ${ }^{7}$ They can extend beyond the costal margin, into the chest wall, and may disrupt the insertion of the diaphragm and violate the separation between the thoracic and abdominal cavities. These defects can extend caudally to involve the inguinal ligament or even the iliac crest as seen in hemipelvectomy resections. Paramedian defects can extend medially beyond the line semilunaris and may involve portion of the ventral abdominal wall. In certain cases, large parastomal hernias can extend laterally to involve a significant portion of the lateral abdominal wall. Posteriorly, defects classified as paraspinal defects can involve portion of the paraspinal musculature and can be associated with formation of Grynfeltt or Petit hernias. Grynfeltt hernias occur within the superior lumbar triangular space formed by the posterior border of the internal oblique muscle, lateral border of the paraspinous muscle, and the 12th rib superiorly. Petit hernias occur more inferiorly and are located within an upright triangular space formed by the posterior border of the external oblique, anterior border of the latissimus dorsi, and iliac crest inferiorly. ${ }^{9}$

\section{Components}

The tissues within the lateral abdominal wall contain a larger ratio of muscle to aponeurotic tissue than the ventral abdominal wall. This reduces the effective tensile strength of the lateral abdominal wall and may even lead to less secure mesh suture fixation in cases of patch-type repairs.

The higher ratio of muscle tissue makes the lateral abdominal wall more susceptible to the consequences of denervation injuries. The oblique muscle complexes are innervated by spinal nerves from T7 to T12 that travel from posterior to anterior in a plane between the internal oblique muscles and transversus abdominis muscles. Defects or denervation injury can affect muscles that are not only directly involved in the resection but can also weaken muscle more medially leading to progressive bulge and hernia formation. ${ }^{7}$

Defects of the ventral abdominal wall are generally bounded by the linea semilunaris laterally and are therefore subject to symmetric forces generated by the oblique muscle complexes. Lateral abdominal wall hernias, however, are subject to significant asymmetric forces. The rectus complexes and contralateral oblique complexes contract in unison on one side of the defect while the ipsilateral oblique if still present and innervated exert weaker forces on the opposite side. The two sides contract independent of each other leading to asymmetric distribution of forces and resultant enlargement or recurrence of hernia or bulge. ${ }^{7}$
Fascial coaptation is also less frequently achieved in lateral abdominal wall repairs. During ventral abdominal wall reconstruction, anterior or posterior component releases can be performed, which can significantly mobilize the rectus complexes to the midline, reduce tension on the fascial closure, and increase the probability of primary fascial coaptation. The muscles around the boundaries of lateral abdominal wall defects cannot be released in a similar fashion, and therefore these defects frequently require bridged repair. If a patch type repair is performed in this setting, it can lead to progressive hernia or bulge recurrence because it does not compensate for the progressive muscle denervation effects. ${ }^{7}$

\section{Management}

\section{Operative Technique}

Lateral abdominal wall reconstruction is generally performed in the setting of hernia or bulge or an excisional defect. The patient is generally placed in a lateral decubitus position on an operating room table that can be appropriately retroflexed or flexed to allow for improved defect exposure and to reduce tension while completing the repair. Multiple techniques for repair and mesh placement have been described. A commonly used technique is a direct mesh or patch repair of the defect. In this case, undermining is performed circumferentially for $5 \mathrm{~cm}$ or until healthy muscle is reached. The mesh is then placed as an underlay (preperitoneal plane) or inlay (between the external and internal oblique muscles) and anchored to the adjacent muscle tissue after removing any redundancy. If possible, the layers of muscle are approximated over the mesh. Proponents of this technique consider it superior to a more extensive pillaranchored repair because they feel that this technique is more synchronous with the dynamic nature of the lateral abdominal wall. They also claim that static repairs such as the pillaranchored repair (described later) have higher local tension at the suture interface because they do not account for muscle compliance. ${ }^{6}$ While reported outcomes for this technique in the literature demonstrate a low recurrence rate, the size of the patient population in these studies is generally small and the defects are not as extensive as those seen with tumor excision surgery.

Another type of repair, described as a pillar-anchored repair, requires anchoring mesh to static boundaries of the abdominal wall. A large piece of mesh is used to resurface the entire lateral abdominal wall. The mesh is anchored to the costal margins superiorly and iliac crest inferiorly using periosteal suture fixation or suture passage through osseous drill holes ( - Fig. 1). ${ }^{7}$ Bone-anchored devices can also be used to fixate suture and mesh. ${ }^{10}$ Anteriorly, mesh is sutured to the linea semilunaris and posteriorly it is sutured to the fascial confluence along the lateral border of the paraspinous muscles. A static repair such as a pillar-anchored repair is expected to have a better outcome profile with respect to hernia or bulge recurrence because it accounts for the disproportionate forces on the abdominal wall and the progressive impact of muscle denervation. A second layer 

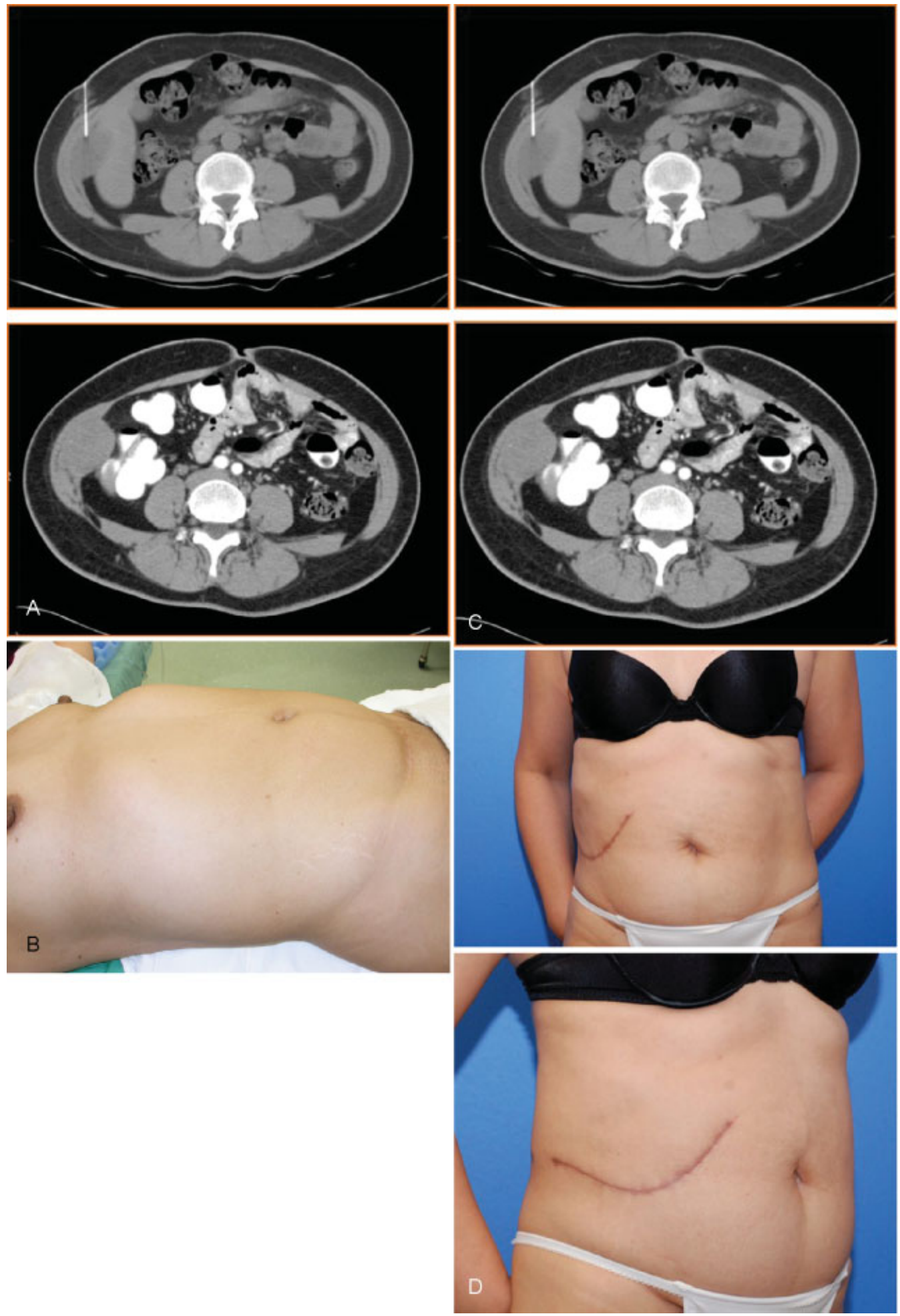

Fig. 1 A 46-year-old woman with desmoid tumor involving full thickness of the lateral abdominal wall. (A) Computed tomography (CT) scans demonstrating the tumor involving the lateral abdominal wall. (B) Anticipated area of resection. (C) 6-month postoperative CT scans demonstrating lack of hernia or bulge. (D) 6-month follow-up pictures demonstrating lack of hernia following reconstruction of lateral abdominal wall with underlay mesh and soft tissue reconstructing with advancement flaps.

of fascial coaptation is not always possible to share the load or tension, which makes a load-bearing approach more necessary. A pillar-anchored approach, however, does require significant undermining and creation of large flaps so that the static boundaries can be visualized and accessed.
The need for pillar-anchored repair is further amplified when the defect crosses boundaries of the abdominal wall. When the defect extends anterior to the linea semilunaris, the mesh is extended to involve the ventral abdominal wall and is anchored along the linea alba in an underlay fashion. A 
contralateral component separation can be performed to reduce tension on the defect. A defect that extends superiorly beyond the costal margin and into the chest wall may involve disruption of the diaphragm attachment and may violate the boundaries between the thoracic and abdominal cavities. In these cases, it is necessary to repair the diaphragm and separate the two cavities. Small defects of the diaphragm can be repaired directly to the edge of the remaining rib but larger repairs may involve elevation of the diaphragm and coaptation to the available external musculoskeletal abdominal wall. In both of these cases, the repair needs to be reinforced with underlay mesh. The mesh is extended superiorly to lie against the peritoneal base of the diaphragm.
Circumcostal or transcostal sutures are then passed around or through the last available rib, through the diaphragm and mesh composite to bolster the repair. Therefore, the mesh effectively helps separate the thoracic and abdominal cavities while bolstering the lateral abdominal wall (-Fig. 2).

Defects that extend posteriorly may include paraspinous muscles, quadratus lumborum, and iliopsoas muscles. They can also include the retroperitoneal reflection and may lead to solid organ herniation. Successful repairs require restoration of the continuity of the muscle layers with simultaneous reinforcement with mesh. The presence of vital structure such as the spinal column, aorta, and vena cava can add to the complexity of the operation and make an underlay
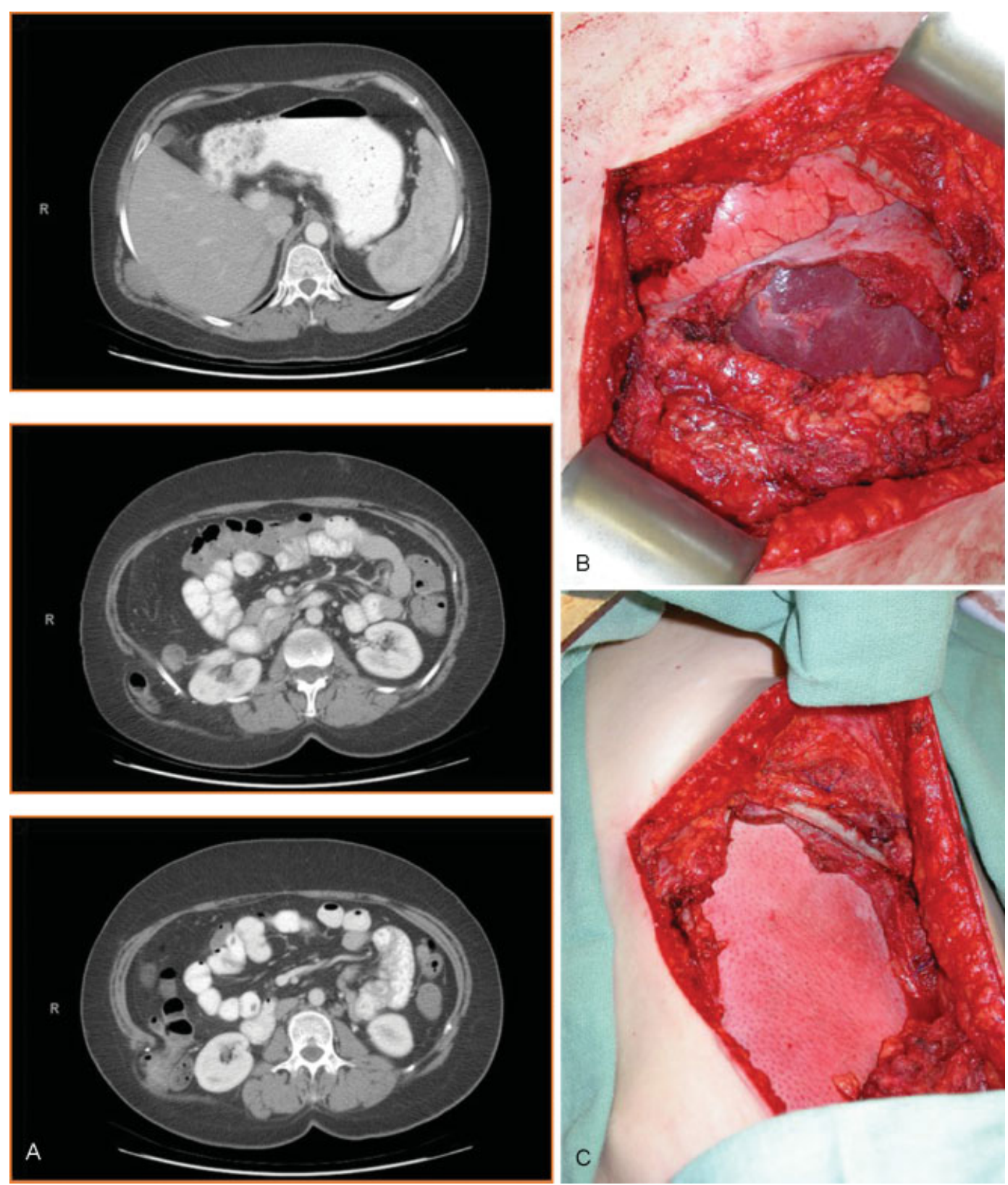

Fig. 2 A 58-year-old woman with recurrent liposarcoma and symptomatic flank hernia who underwent radical resection and hernia repair. (A) Computed tomography (CT) scans demonstrating the tumor involving the lateral abdominal wall. (B) Composite thoracoabdominal wall resection including rib 9 to rib 12 . (C) Reconstruction using underlay mesh placement. (D) 6-month postoperative CT scans demonstrating healed repair without hernia or bulge formation. (E) 6-month follow-up oblique and lateral views. 

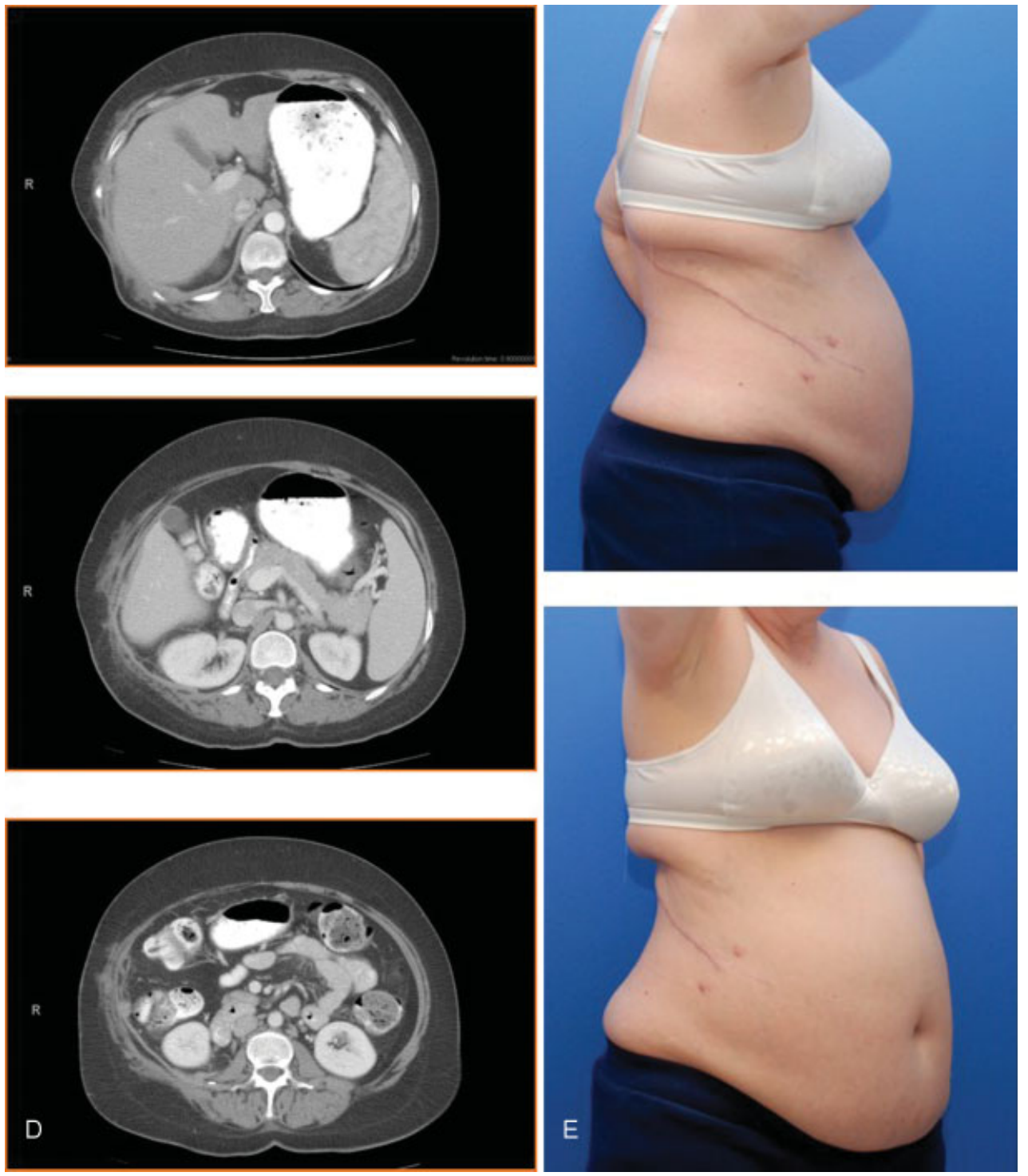

Fig. 2 (Continued)

placement of mesh challenging or impossible. In these cases, the mesh is placed in an onlay fashion.

Extensive abdominal wall defects are also noted in the setting of hemipelvectomy surgery performed for resection of certain tumors of the pelvis and lower extremities. The extent of resection is classified based on the part of the pelvis that is resected. Majority of the caudal support for the lateral abdominal is based on the iliac crest. When portion or all of the ilium is resected, an extensive piece of mesh is required to reconstruct the abdominal wall and is anchored to the remaining osseous support in the pelvis. ${ }^{11}$

\section{Choice of Mesh Utilization}

As described earlier, successful reconstruction of lateral abdominal wall defects is highly reliant on a load bearing mesh support. The mesh size is generally extensive, and in certain cases, the mesh serves as an interposition or bridged repair when overlying muscle layers cannot be primarily closed. Important considerations behind the choice of mesh include long-term strength and stability, reduction in risk of surgical site infections, reduction in adhesion formation to internal organs, and reduction in risk of erosion and fistula formation. The operative setting including patient comorbidities, wound contamination, and availability of omentum to shield internal organs must also be taken into account while choosing between mesh materials. Synthetic meshes are cheaper and heal with scar tissue formation or encapsulation. They can lead to an increased risk of surgical site occurrences in contaminated fields. Bioprosthetic mesh materials, while being more expensive, have the advantage of healing by tissue regeneration and revascularization, leading to a low infection risk. These mesh materials are also associated with a lower risk of adhesion and fistula formation. In the event of mesh exposure, they can be managed with local wound care in most cases, thereby avoiding the morbidity of mesh explantation. Furthermore, the ability of bioprosthetic mesh to integrate with native tissue is beneficial at the time of reoperation. In many cases, the mesh can be incised and later reapproximated analogous to primary fascia. ${ }^{7,12,13}$ 


\section{Soft Tissue Reconstruction}

Durable, tension-free, soft tissue closure is imperative to reduce the risk of mesh exposure, obliteration of dead space, and prevention of seroma or fluid collection. In the absence of prior radiation or multiple surgical incisions, large wellperfused soft tissue flaps can be elevated and advanced. If needed, rotation and advancement flaps can also be designed. If the defect is large, thoracoepigastric bipedicled flaps can be designed and advanced into defect.

In the setting of poor tissue quality or prior radiation, pedicled flaps can be brought in to resurface the defect. In the upper abdomen, these options include vertical rectus abdominis myocutaneous flaps (VRAM), latissimus dorsi myocutaneous flaps, or omental flaps. Inferiorly positioned defects can be reconstructed with thigh-based flaps such as pedicled anterolateral thigh flaps, vastus lateralis flaps, or tensor fascial lata flaps. If the defect cannot be reached or resurfaced by pedicled flaps, free tissue transfer should be considered. Recipient vessels for flap transfer include deep inferior epigastric vessels, internal mammary vessels, superior epigastric vessels, thoracodorsal vessels, and thoracolumbar and intercostal artery perforators. Vein grafts to these vessels may be required if the pedicle does not have adequate reach. $^{7}$

\section{Conclusion}

Lateral abdominal wall defects vary significantly from the more commonly occurring ventral defects. The lateral abdominal wall has a different composition and is subject to a different set of forces, which require careful attention when planning the repair. There is a higher proportion of muscle to aponeurosis; consequently, muscle denervation plays a more significant role. The forces along the borders of the defect are asymmetric and unbalanced leading to increased risk of progression and recurrence. Since this portion of the abdominal wall is more dynamic, a stable repair requires attachment of mesh to the static pillars of the abdominal wall. Primary fascial coaptation may not be as easily attained because there is no analogous, tension-reducing fascial or component release that can be performed. While there are many proponents of both patch type repair and pillar-anchored repair techniques, a formal comparison of the two techniques is lacking. Bioprosthetic mesh is preferred over synthetic mesh to reduce the rate of infection and adhesion formation. Durable soft tissue coverage is important to protect the mesh repair. Soft tissue reconstruction may require a simple advancement flap, a pedicled flap, or a free tissue transfer.

\section{Acknowledgments}

The author thanks Dr. Donald Baumann in the Department of Plastic Surgery, The University of Texas MD Anderson Cancer Center, Houston, Texas, who provided figures used in this chapter.

\section{References}

1 Fakhry SM, Azizkhan RG. Observations and current operative management of congenital lumbar hernias during infancy. Surg Gynecol Obstet 1991;172(06):475-479

2 Chatterjee S, Nam R, Fleshner N, Klotz L. Permanent flank bulge is a consequence of flank incision for radical nephrectomy in one half of patients. Urol Oncol 2004;22(01):36-39

3 Matsen SL, Krosnick TA, Roseborough GS, et al. Preoperative and intraoperative determinants of incisional bulge following retroperitoneal aortic repair. Ann Vasc Surg 2006;20(02): 183-187

4 Nanni G, Tondolo V, Citterio F, et al. Comparison of oblique versus hockey-stick surgical incision for kidney transplantation. Transplant Proc 2005;37(06):2479-2481

5 Gardner GP, Josephs LG, Rosca M, Rich J, Woodson J, Menzoian JO. The retroperitoneal incision. An evaluation of postoperative flank 'bulge'. Arch Surg 1994;129(07):753-756

6 Purnell CA, Park E, Turin SY, Dumanian GA. Postoperative flank defects, hernias, and bulges: a reliable method for repair. Plast Reconstr Surg 2016;137(03):994-1001

7 Baumann DP, Butler CE. Lateral abdominal wall reconstruction. Semin Plast Surg 2012;26(01):40-48

8 Pezeshk RA, Pulikkottil BJ, Bailey SH, et al. An evidence based model for the successful treatment of flank and lateral abdominal wall hernias. Plast Reconstr Surg 2015;136(02):377-385

9 Salameh JR, Salloum EJ. Lumbar incisional hernias: diagnostic and management dilemma. JSLS 2004;8(04):391-394

10 Elkwood AI, Kozusko SD, Patel TR, et al. The bony anchoring reinforcement system (BARS) for flank hernia repair: a versatile technique. Eur J Plast Surg 2017;40:315-322

11 Chao AH, Neimanis SA, Chang DW, Lewis VO, Hanasono MM. Reconstruction after internal hemipelvectomy: outcomes and reconstructive algorithm. Ann Plast Surg 2015;74(03):342-349

12 Butler CE. The role of bioprosthetics in abdominal wall reconstruction. Clin Plast Surg 2006;33(02):199-211, v-vi,v-vi

13 Jin J, Rosen MJ, Blatnik J, et al. Use of acellular dermal matrix for complicated ventral hernia repair: does technique affect outcomes? J Am Coll Surg 2007;205(05):654-660 